\title{
Approaches for implementation and updation of community health needs assessment: an updated review
}

\author{
Kalidas D. Chavan ${ }^{1 *}$, Purushottam A. Giri ${ }^{2}$, Savita Rajurkar ${ }^{3}$, Satish D. Pawar ${ }^{4}$
}

\author{
${ }^{1}$ Registrar, Maharashtra University of Health Sciences (MUHS), Nashik, Maharashtra, India \\ ${ }^{2}$ Professor and Head, Department of Community Medicine, IIMSR Medical College, Badnapur, Jalna, Maharashtra, \\ India \\ ${ }^{3}$ Chair, Lead faculty, Research Investigator, (Helfgott Reserach Institute), Ayurved Program, National University of \\ Natural Medicine (NUNM), USA \\ ${ }^{4}$ Additional Mission Director (National Health Mission), Public Health Department, Govt. of Maharashtra, Mumbai, \\ Maharashtra, India
}

Received: 16 November 2018

Revised: 01 December 2018

Accepted: 03 December 2018

\author{
*Correspondence: \\ Dr. Kalidas D. Chavan, \\ E-mail: kdchavan17@gmail.com
}

Copyright: $(\odot$ the author(s), publisher and licensee Medip Academy. This is an open-access article distributed under the terms of the Creative Commons Attribution Non-Commercial License, which permits unrestricted non-commercial use, distribution, and reproduction in any medium, provided the original work is properly cited.

\begin{abstract}
Implementation of assessment is utmost significant to enhance the prospects and strengths of Community Health Needs. Addressing community health needs has been a challenge for the Governments of various nations in the world due to various reasons like rising number of recipients, insufficient resources, increase in demands of resources and discrimination in their supply. The role of community health assessment is central in enabling practitioners, managers and policy makers to identify, categorize and prioritize demands, update the policies and then, to ensure that these health care resources are supplied to optimize health care and maximize its outreach to community members. It has a great potential to be a vital tool which can empower the management and planning of health care across the countries at the level of nationwide, state-wide and district wide communities, populations and families. The objective of this review article is to present the systematic and updated approaches to implement community health needs assessment at multiple levels i.e. district, state and national, keeping those aligned with the approaches indicated within global policies. It's measurable outcomes are to identify and list the priority health needs, target resources to address inequalities, involve local people, educate and train the stakeholders and provide evidence based updated data for amending existing policies. The process of undertaking community health needs assessment and the importance of contribution of health care personnel in this process is also included here.
\end{abstract}

Keywords: Community health, Needs assessment, Health care, Approach for implementation \& updation

\section{INTRODUCTION}

Community Health Needs Assessment (CHNA) is defined as a systematic process involving the community to identify and analyse community health needs and assets, prioritize the needs, and implement a plan to address significant unmet community health needs. A community health needs assessment is not a 'one-off' activity but an on-going developmental process and can be amended, over the time. It is not just about looking for problems and needs but also about assessing the strengths and resources/assets that promote wellbeing in the community. Communities are typically defined by a geographic area. However, they can also be based on shared interests or characteristics such as religion, race, age, values, occupation and income. People within a community come from different backgrounds and have 
unique identity with respect to cultures, customs, and values. It is not an end in itself but provides an outlook and considerably valuable information to plan health care and public health programmes in the future. ${ }^{1,2}$

\section{Objectives and outcomes}

The objectives of this review article are to present the systematic and updated approaches to implement community health needs assessment at multiple levels viz. district, state \& national, keeping those aligned with the approaches indicated within global policies. Propose the idea of 'policy updation' in the context of updated evidence based data by comparing real time data with the previous data, by reviewing the existing policies which were set up in relevance with the previous data, community health need assessments records and conditions. It's measurable outcomes are to identify and list the priority health needs, target resources to address inequalities, involve local people, educate and train the stakeholders, and provide evidence based updated data for amending existing policies. The process of undertaking community health needs assessment and the importances of contribution of health care personnel in this process are also included here. Current article is an attempt to review various community health needs, and applicable solutions which are aimed to prevent diseases and improve the health of recipients.

\section{METHODS OF LITERATURE SEARCH}

Medical Subject Headings of electronic databases and internet search engines PubMed, PubMed Central, Google Scholar, Medknow, Science Direct and Textbooks until 2017 were searched as review materials. Authentic Literatures on the 'community health needs assessment' in the form of policies, research papers, reports, books published by health authorities, government, national \& international agencies \& associations in print \& electronic format were retrieved. The key words used for the literature search included "community health" and "needs assessment". Metaanalyses and multi country study reports compiled in English language were retrieved and evaluated from year 1990 to 2017. A total thirty five relevant authentic articles were identified. After examining the abstracts, contents, and subject matter, the significant twenty five articles were considered for this review.

\section{CONCEPTS AND PRINCIPLES OF HEALTH NEEDS ASSESSMENT}

According to World Health Organization (WHO), Health is a state of complete physical, mental and social wellbeing and not merely the absence of disease or infirmity. ${ }^{3}$ This review uses a holistic model of health, emphasizing the social, economic and cultural factors that affect health as well as individual behaviour. Community health refers to meeting the needs of a community by identifying their health problems and managing the well-being of members of the community. Community health, is a field of public health, is a discipline that concerns itself with the study and betterment of the health characteristics of biological communities. ${ }^{4,5}$ The concept of "need" used in this review incorporates those needs felt and expressed by local people as well as those defined by professionals. It moves beyond the concept of demand and takes account of people's capacity to benefit from health care and public health programmes.

\section{FACTORS AFFECTING HEALTH}

Health is affected by a number of factors, viz. the physical environment in which people live, such as the quality of the air they breathe and the water they drink; the social environment is the level of social and emotional support people receive from friends and/or family; poverty, a significant factor worldwide, which shortens and reduces enjoyment of life; behaviour and lifestyle, for example, smoking causes lung cancer and coronary heart disease so a reduction in this behaviour will reduce the disease; and family genetics and individual biology if you come from a healthy family then you have a better chance of health and wellbeing. ${ }^{6}$

\section{LOCAL PEOPLE'S VIEWS OF THEIR HEALTH NEEDS AND HEALTH SERVICES}

A central part of health needs assessment is gathering information on local people's views of their health needs and resources. Involving local people will ensure that any service developed will be based on need and will be more likely to be acceptable to the population. There will always be a great deal of expertise and knowledge to draw on among the local population, in particular on what assets exist, the factors that influence their health, what is most important, local health beliefs and probable solutions to their health problems. It is important that people are able to describe health problems and solutions in their own words. This may involve using less conventional methods such as photographs, drawings or personal stories. Be flexible and ask the community which methods are most suitable. The community view or perspective is important to include, and involving local people initiates their involvement and participation in improving the health of their own community. ${ }^{7,8}$

\section{WHAT IS THE GOAL OF COMMUNITY HEALTH NEEDS ASSESSMENT?}

Needs assessment will enable us to plan and deliver the most effective care to those who are in the greatest need; apply the principles of equity and social justice in practice; ensure that scarce resources are allocated where they can give maximum health benefit; work collaboratively with the community, other professionals and agencies to determine which health issues cause greatest concern and plan interventions to address those issues. ${ }^{8,9}$ 


\section{WHAT IS THE OUTCOME OF 'COMMUNITY NEEDS ASSESSMENT' IN GENERAL?}

A community needs assessment provides a snapshot of local policies, systems, and current strategies in environmental change. It helps to identify areas for improvement. On the basis of this data, a course for health improvement can be mapped out for setting up strategies to make positive and sustainable changes in their communities. ${ }^{9,10}$

\section{WHAT ARE THE OUTCOMES OF COMMUNITY HEALTH NEEDS ASSESSMENT?}

Community Health Needs Assessment provides baseline information about the health status of recipients from the community. The assessment ensures that decisions are based on solid information and evidence; helps set priorities; helps regional health authorities to assess outcomes and results in the longer term; gets community members, stakeholders and a wide variety of partners involved in the decision-making process, helps them understand the difficult choices that need to be made, and builds support and commitment for addressing health needs on a community-wide basis. It can provide a benchmark information as a base for the funding allocations; can be used to guide policy and program development; can assist in mapping out links and interdependency of other sectors; can provide insights into the fundamental causes and pathways of diseases and ill-health, and provide population-based information to identify opportunities for disease prevention, health promotion and health protection. ${ }^{10-12}$

\section{COMPONENTS OF OUTCOMES OF A COMMUNITY HEALTH NEEDS ASSESSMENT ${ }^{13-16}$}

The main outcomes of a community needs assessment are measured in three prime categories: Policy change, Systems change, and Environmental change.

\section{Policy change}

This involves laws, regulations, rules, protocols, and procedures that are designed to guide or influence behaviour of community members. Policies can be either legislative or organizational. Policies often mandate environmental changes and increase the likelihood that they will become institutionalized or sustainable. Examples of legislative policies include taxes on tobacco products, provision of county or city public land for green spaces or farmers' markets, regulations governing a national school lunch program, and clean indoor air laws. Examples of organizational policies include schools requiring healthy food options for all students, menu labelling in restaurants, and required quality assurance protocols or practices (e.g., clinical care processes) etc.

\section{System change}

This involves 'the change' that affects all community components including social norms of an organization, institution or system. It may include a policy or environmental change strategy. Policies are often the driving force behind systems change. Examples are implementing a national school lunch program across a region or provincial school system or ensuring a hospital system becomes tobacco free etc.

\section{Environmental change}

This type of change relates to the physical, social, and/or economic factors designed to influence people's practices and behaviours.

Examples of alterations or changes to the environment include below factors:

Physical: Structural changes or the presence of programs or services, that include the presence of healthy food choices in restaurants or cafeterias, improvements in the in-built environment to promote walking, jogging (e.g. walking tracks, jogging tracks), the availability of smoking cessation services to patients or workers, and the presence of comprehensive school health education curricula in schools.

Social: A positive change in attitudes or behaviour about policies that promote, viz. health, and/or an increase in supportive attitudes regarding health care practices, an increase in favourable attitudes of community decision makers about the importance of non-smoking policies and/or an increase in non-acceptance of exposure to second-hand smoke from the general public.

Economic: The presence of financial disincentives for arresting undesired and unhealthy behaviours and/ or incentives to encourage a desired behaviour of community members. For example, include applying higher prices for tobacco products to decrease their use or the provision of discounts in health insurance for nonsmoking applicants.

\section{HOW DO YOU PREPARE FOR A COMMUNITY HEALTH NEEDS ASSESSMENT? ${ }^{17-20}$}

Assessing community health needs is an essential component of the health planning cycle. It is important that decisions and policies are grounded in solid information about the health needs of community members regardless of the variability in approaches of health planning cycle. Realization of the significance of assessing community needs is an essential factor. However, actual undertaking and implementing this process is a complex, time-consuming job and a doable hard work. 
Concerned manifold discussions and actions are enumerated below.

- Community health needs assessment is a big task which demands time, manpower and resources. To begin with, a regular regional health planning cycle can be set up. Since community health needs a 'do not change rapidly' approach, plan on doing an initial comprehensive health needs assessment, an annual scan of your environment, and then an ongoing comprehensive health needs assessments about every three years, preferably as part of the regular regional health planning cycle.

- Build on expertise in your region but also plan to involve due outside expertise as per requirement. Build on the skills, resources and information that already exist in your organization and communities. In view of the integral part the community health needs assessment which will play a vital role in the overall regional health planning process, regional health authorities should seriously consider setting up of a responsibility centre within their region.

- Community health needs assessments can be done at different levels. In addition to a broad and comprehensive assessment, more in-depth work should be done to target a particular issue in the community or develop a program response for a particular consumer group. This can provide a clearer picture of the problem and possible solutions.

- An extensive data can be available to get collected, but it can incur unreasonable costs and time. It could be worth concentrating on the entitled key data that can allow your region to track important issues, trends and results.

- It is required to distinguish between "needs" and "wants". Identify each one to prioritize accordingly. For example, to prevent outbreaks of communicable diseases in children, the clearly indicated "need" is to focus on immunization of the children. On the other hand, "wants" are things we often think will solve a problem or meet a need. They are just one of the many ways of approaching a problem and they may not necessarily prevent that problem from occurring or address a basic need. For example, elderly people who can no longer manage alone "need" a safe, supportive living environment. The community may "want" a new long term care facility for elderly people. However, an expanded community care or assisted living may be a better alternative and more appropriately meet the "need". A community health needs assessment provides an opportunity to educate and involve community members and consumers in making choices about new ways to meet due needs.

\section{STEPS OF COMMUNITY HEALTH NEEDS ASSESSMENT ARE AS FOLLOW ${ }^{21-23}$}

- Profiling- Involves the collection of relevant information that will inform the nurse about the state of health and health needs of the population; and analysis of this information to identify the major health issues.

- Deciding on priorities for action

- Planning public health and health care programmes to address the priority issues

- Implementing the planned activities

- Evaluation of measurable health outcomes

\section{RESEARCH COLLABORATION}

By incorporating community-based research and patientcentred research principles, the CHNA process, hospitals and health systems can develop an approach that promotes sustainable community engagement and information exchange. Furthermore, knowing how existing research findings can be applied to address identified health needs in the community, which has the potential to increase the impact and efficacy of interventions. Strategically applying research principles and results to the CHNA process can help hospitals and health systems achieve their goal of fostering a healthier community where all individuals can reach their highest potential for health. ${ }^{24}$

\section{THE NEED OF 'COMMUNITY HEALTH NEEDS ASSESSMENT'}

The costs of healthcare are rising. Over the past thirty years expenditure on health care has risen much faster than the cost increases reported in other sectors of the economy, and health care is now one of the largest sectors in most developed countries. Medical advances and demographic changes will continue the upward pressure on costs. At the same time the resources available for health care are limited. Many people have inequitable access to adequate health care, and many governments are unable to provide such care universally. In addition there is a large variation in availability and use of health care by geographical area and point of provision. Availability tends to be inversely related to the need of the population served. ${ }^{4}$

The example of Maharashtra state is remarkably applicable and acceptable here as an evidence. This state has progressed well in many of the health indicators. Within the state there are inter-district and intra-district variations in health indicators. There is vast discrepancy in the spread of health services among the districts of Maharashtra. It is necessary to emphasis on the increase in infrastructure in rural area. The local governments i.e. Zila Parishad and Gram Panchayat seem to be indifferent to health expenditure and health status of the people in rural area. State Governments can proactively focus on optimum use and extend funds allocated in the budget for public health sector. The rural public health services are facing the problem of inadequate manpower e.g. doctors, paramedical staff and other staff and it has direct influence on the utilisation of rural public health centers by the rural people. The private and corporate health services are not affordable for poor and downtrodden 
rural mass. Hence, the government should take steps to reduce this shortfall of manpower as well as emphasis on increase in health investment in rural area. As there are number graduates and post graduate and qualified doctors and nurses available in the state, they may be employed at the shortfall posts. But government is shifting its responsibility to NGO and to private sector, but it is not desirable. The constitutional provisions regarding health services seem to be forgotten by the state Government. ${ }^{4,23}$

\section{LOCAL AND NATIONAL PRIORITIES}

National priorities are often set by governments and influenced by the political and economic agenda. Local priorities reflect national priorities as well as issues identified by local groups, practitioners and communities. A fundamental requirement while undertaking a community health needs assessment is to discover these priorities in relation to health. Sometimes there can be a conflict between the national top-down agenda and locally identified needs. ${ }^{15-19}$

Maharashtra state from India is used as an evidence and considerably acceptable example in this article because Maharashtra state has been in the forefront of healthcare development in India. It was among the first few states who took steps to decentralize primary healthcare administration through Zilla Parishads (District council) as early as in 1961. Maharashtra was one of the first states to achieve the mandated norms for primary health centres, sub-centres and Rural Hospitals. The state also has the largest private health sector of India with the remarkably extensive outreach. Despite being the most affluent state in the country showing up the highest per capita income, contributing over $15 \%$ of the country's national income and $40 \%$ of the incoming tax revenue as of today, Maharashtra continues to have high levels of poverty and inequalities. The reflections of inequality and variable financial status of regions are not commendable on the health outcomes. Maharashtra has to still struggle in the areas like malnutrition deaths, child mortality, noncommensurability of the maternal mortality levels with its economic position in the country, declining child sexratios, low and declining levels of public health spending and investments, high levels of vacant positions of doctors at PHCs and CHCs, and low levels of access to various health services like antenatal care, complete child immunization, institutional deliveries etc. ${ }^{4}$

Graduates of modern medicine provide health care through a practice of modern medicine in their own field as a generalist or specialist doctor. A health care is provided by large group of graduates from integrated medicine/ traditional and complementary medicine like Ayurved, Yoga, Unani, Siddha, Homeopathy, Chinese Medicine, Naturopathic Medicine professionals. Some of them practice as general practitioners in their own field, or integrate the practices of modern medicine or adapt only modern medicine in practice. There should be clear guidelines for all of them regarding the threshold conditions which would call for an allopathic medicine. They should be educated to understand as to 'when' they can treat the patients in their relevant field and when they need to refer the patient to an allopathic generalist or specialist doctor. ${ }^{20,21}$ Therefore, those competencies should be targeted in their curriculum. Competency oriented curricula should be taught, assessed and certified. Some of the defined competencies as per millennium development goals should be mandatory to incorporate in practice for any general practitioner including integrated/ traditional medicine practitioners.

\section{INDIA'S OUTSTANDING APPROACH TO ENHANCE HEALTH}

\section{National health policy 2017}

Government of India has a special emphasis on Community participation in health planning processes. 6 NHP advocates increase community health volunteers to population ratio as per Indian Public Health Standard IPHS norm. Promotion of healthy living and prevention strategies by incorporating healthcare modalities from AYUSH systems, viz. Ayurved, Yoga, Natural Medicine, Unani, Siddha, Homeopathy. For example, at the personal and family level, diurnal, seasonal regimen from Ayurved, and at the level of workplace, schools and community group activities, Yoga would also be an important form of health promotion that has a special appeal and acceptability in the Indian context. This approach is getting an increasing popularity in global settings. For better public health support from local selfgovernment and the Village Health Sanitation and Nutrition Committee (VHSNC) is essential. Better secondary prevention would also be an integral part of an urban health strategy. Improved health seeking behaviour, influenced through capacity building of the community based organizations \& establishment of an appropriate referral mechanism, would also be the important components of this strategy. Noncommunicable diseases (NCDs) like hypertension, diabetes which are predominant in the urban areas would be addressed under NUHM, through planned early detection. There is a need to halt and reverse the growing incidence of chronic diseases. The policy recommends to set-up a National Institute of Chronic Diseases including Trauma, to generate evidence for adopting cost effective approaches and to showcase best practices. This policy will support an integrated approach where screening for the most prevalent NCDs with secondary prevention would make a significant impact on reduction of morbidity and preventable mortality. This would be incorporated into the comprehensive primary health care network with linkages to specialist consultations and follow up at the primary level. ${ }^{23-25}$

\section{CONCLUSION}

This review has outlined a process to assess community health needs and to use that information to set priorities, 
choose and execute appropriate actions, and assess results. Requirement of reviewing the existing policies in the light of updated data and results and incorporate due amendments to set up updated policies can help enhance the outcomes and goal of community health needs assessment. Health needs assessment is a multidisciplinary and multi-sectoral activity. A thorough and comprehensive community health needs assessment process will ensure that each regional health authority has a solid base of information about the needs, capacities and expectations of communities. It ensures that you are, in fact, responding to the most pressing needs and listening to your communities and that your regional health plan addresses community health needs.

Funding: No funding sources

Conflict of interest: None declared

Ethical approval: Not required

\section{REFERENCES}

1. Centres for Disease Control and Prevention. Community Health Assessment and Group Evaluation (CHANGE) Action Guide: Building a Foundation of Knowledge to Prioritize Community Needs. Atlanta: U.S. Department of Health and Human Services, 2010.

2. Alberta Health, Prevention and Promotion Branch. Assessing Community Health Needs: A Guide for Regional Health Authorities. 2nd edition. 1996.

3. Park K. Park's Textbook of Preventive and Social Medicine, Concept of Health and Disease. 23rd edn. M/s Banarsidas Bhanot Publishers; 2015: 22.

4. Health Planning In Maharashtra State, Chapter three, 2010.

5. Wright J, Williams R, Wilkinson JR. Health needs assessment, Development and importance of health needs assessment, BMJ. 1998;316:25.

6. Alberta Health: Assessing Community Health Needs An Overview for Regional Health Authority Boards. January, 1995.

7. South Australian Community Health Research Unit. Planning Healthy Communities: A Guide to Doing Community Needs Assessment. 1990.

8. Community Needs Assessment. Atlanta, GA: Centers for Disease Control and Prevention (CDC). 2013.

9. Community Health Assessment and Group Evaluation (CHANGE) data-collection tool developed by CDC's Healthy Communities Program in the Division of Community Health at the National Center for Chronic Disease Prevention and Health Promotion. Available at: http://www.cdc. gov/HealthyCommunitiesProgram. Accessed on 12 November 2017.

10. Community Assessment Guide Book, North Carolina Department of Health (2002); Available at:http://www.schs.state.nc.us/schs/data/databook/20
02/GuideBook2002.pdf. Accessed on 19 October 2012.

11. Centers for Disease Control and Prevention (CDC). Healthier Worksite Initiative, Environmental Audits. Available at: http://www.cdc.gov/nccdphp/ dnpao/hwi/programdesign/environmental_audits.ht m. Accessed on 19 October 2016.

12. Prince Edward Island. Health and Community Services System. Community Needs Assessment: The Prince Edward Island Experience. November, 1995.

13. Department of Health and Social Services. Government of the Northwest Territories, Working Together for Community Wellness: A Directions Document. April, 1995.

14. Voluntary Hospitals of America, Inc., Community Health Assessment: A Process for Positive Change. 1993.

15. Wright J. Health needs assessment in practice. London: BMJ Books; 1998.

16. Robinson J, Eklan R. Health needs assessment: theory and practice. London: Churchill Livingstone; 1996.

17. Hooper J, Longworth P. Health needs assessment in primary care: a workbook for primary health care teams. Calderdale and Kirklees Health Authority, 1997.

18. Thomas DN. In: Freeman R. et al., ed. Community development and involvement in primary care. London: King's Fund; 1995.

19. HEALTH21. The health for all policy frame work for the WHO European Region. Copenhagen, WHO Regional Office for Europe, (European Health for All Series, No. 6) 1999.

20. Bowling A. Measuring health: a review of quality of life measurement scales. Buckingham, Open University Press, 1991.

21. Stevens A, Raftery J. Health care needs assessment. Oxford: Radcliffe Medical Press; 1994.

22. Appleton J, Cowley S, ed. The search for health needs. Basingstoke: Macmillan Press; 2000.

23. National Health Policy - 2017, Department of Health \& Family Welfare, Govt. of India. 2017

24. Rowe A, McClelland A, Billingham K. Community Health Needs Assessment- An introductory guide for the family health nurse in Europe. Institute of General Practice and Primary Care, University of Sheffield, United Kingdom. 2001.

25. Health Research \& Educational Trust. Applying research principles to the community health needs assessment process. Chicago, IL: Health Research \& Educational Trust. Available at: http://www.hope. org. Accessed on 12 July 2017.

Cite this article as: Chavan KD, Giri PA, Rajurkar S, Pawar SD. Approaches for implementation and updation of community health needs assessment: an updated review. Int J Community Med Public Health 2019;6:440-5. 